\title{
Essential RNA-based technologies and their applications in plant functional genomics
}

\section{Sachin Teotia ${ }^{1,2,3}$, Deepali Singh ${ }^{2,3}$, Xiaoqing Tang ${ }^{3}$, and Guiliang Tang ${ }^{1,3}$ *}

${ }^{1}$ Provincial State Key Laboratory of Wheat and Maize Crop Science, Henan Agricultural University, Zhengzhou, 450002, China

${ }^{2}$ School of Biotechnology, Gautam Buddha University, Greater Noida, U.P., 201312, India

${ }^{3}$ Dept. of Biological Sciences, Michigan Technological University, Houghton, MI 49931, U.S.A.

* To whom correspondence should be addressed:

Department of Biological Sciences, Michigan Technological University, Houghton, MI, 49931, U.S.A.

Email: gtang1@mtu.edu

Phone: 906-487-2174

Fax: 906-487-3167

Keywords: Target mimic (TM); short tandem target mimic (STTM); microRNA sponge (SP); artificial microRNA (amiRNA); artificial/synthetic tasiRNA (atasiRNA/syn-tasiRNA); CRISPR/Cas9. 


\begin{abstract}
Genome sequencing has not only extended our understanding of the blueprints of many plant species but also revealed the secrecy of coding and non-coding genes. Here, we present a brief introduction and personal account for a few key RNA-based technologies and their development and applications for functional genomics of plant coding and non-coding genes, with a focus on short tandem target mimic, artificial microRNAs and CRISPR/Cas9. Furthermore, their multiplex technologies are discussed for functional dissection of gene networks.
\end{abstract}

\title{
Approaches for functional genomics studies in plants
}

It has been intriguing to functionally characterize genes in order to understand their distinctive roles in plant development and physiology. Traditional forward genetics approaches, such as positional cloning, involves identifying gene(s) associated with phenotypes [1]. In contrast, reverse genetics, which includes T-DNA insertion and tagging [2], focuses on identifying the function of a given gene. Functional genomics was proposed upon genome sequencing [3], particularly by a large-scale identification of genes through high throughput sequencing [4]. After genes are identified, functional characterization of genes is mainly achieved by creation of various gain- or loss-of-function mutants in plants [5]. Traditional gain of gene functions in plants is achieved through expression of target genes driven by strong promoters like $C a M V 35 S$ or through $C a M V 35 S$ enhancers, such as activation tagging [6]. Loss of gene functions is commonly induced by EMS or T-DNA/transposon insertion [7-10], which leads to mutated/truncated proteins with attenuated or null functions. All these technologies are targeting genes in a random manner without any specificity.

The later technologies are aimed at gene targeting to induce loss-of-function. These losses of functions can be achieved by either targeting DNA or RNA of a specific gene for alterations or silencing. To target specific DNA (gene) in the genome, technologies such as Zinc finger nucleases (ZFNs) [11], transcription activator-like effector nucleases (TALENs) [12], Meganuclease [13], and Clustered Regularly Interspaced Short Palindromic Repeats (CRISPR)associated nuclease 9 (Cas9) (CRISPR/Cas9) have been developed [14, 15]. ZFNs, TALENs, and Meganuclease require complicated protein engineering before altering genes of interest. In contrast, CRISPR/Cas9 is an RNA-based DNA cleavage technology, making its application as simple as RNAi but more directional, effective and diverse than traditional methods for creating genetic mutants [15]. Targeting the RNA of a given gene for silencing involves the use of RNA interference (RNAi), a great discovery in silencing genes, post-transcriptionally [16-18]. Over the years, RNAi technology has undergone many modifications in development, starting from a hairpin structure with inverted repeats to artificial microRNAs [19-22]. RNAi/small RNA-based gene silencing is being overwhelmingly used as a popular means to study gene function because it could target specific genes of known sequences to decipher their functions for the first time in a non-random manner. It can lead to gene silencing at either transcriptional (target DNA methylation) or post-transcriptional level (target RNA cleavage or translational repression) [2325]. In the genomics era when genomes of many plant and animal species have been sequenced, RNAi/small RNA-based gene silencing is extremely useful and has become a powerful approach 
to functional genomics, especially when genetic mutants are unavailable or not feasible (Figure 1).

Small RNAs, including microRNAs (miRNAs) and small interfering RNAs (siRNAs), have emerged as key players in gene regulation during growth and development, epigenetics and responses to various abiotic and biotic stresses by negatively regulating gene expression at the post-transcriptional level [26]. Hundreds to thousands of miRNAs have been identified from different plant species, and there are increasing interests and efforts in miRNA discovery with the advent of high-throughput sequencing and the completion of whole genome sequences [27]. Functional genomics of these small RNA genes in the genome has become a new subject for technology development in plants and animals. Because of their small size, traditional technologies are not well applicable to study functions of small RNAs. Several specific technologies for functional genomics of small RNAs, such as miRNA target mimic (TM) [28], short tandem target mimic (STTM) [29-31], and miRNA sponge (SP) [32], have been developed. These technologies are powerful in inactivating small RNAs at the post-transcriptional level. Similarly, artificial microRNAs (amiRNAs) have been successfully used to downregulate target mRNAs [22] and even miRNAs [33]. Artificial/synthetic trans-acting small interfering RNAs (atasiRNAs/syn-tasiRNAs) can also be used as an alternative to induce specific gene silencing in plants [34]. As a complementation, the newly developed RNA-based CRISPR/Cas9 technology can also be used for editing coding and non-coding genes [35, 36]. It is feasible that such a strategy can be used to knock out multiple miRNAs or miRNA families through a multiplex of guide RNAs (gRNAs), just like targeting multiple coding genes by this approach [37]. The multiplex technology of CRISPR/Cas9 also gives insight into the development of multiplex technology for other small RNA-related technologies. In this article, we focus and discuss on how these RNA-based technologies are developed, applied, and distinguished as essential technologies for plant functional genomics (Figure 1). We also offer insights into the further development and applications of these technologies in plants or even in animals.

\section{Short Tandem Target Mimic (STTM) and related technologies to downregulate small RNAs}

Plant and animal microRNAs normally occur in large families with each family featuring many members [38]. Although different members exhibit mostly distinct temporal and spatial expression patterns, certain levels of functional redundancy are established among the members of a miRNA family [39]. Functions of miRNAs can be studied either by overexpressing them or their modified targets, which are resistant to cleavage by the respective miRNAs. However, neither approach is sufficient to study the functions of miRNAs. Overexpression of a miRNA (gain-of-function of the miRNA) can partially reveal the miRNA functions, but it may not reveal the functions of a miRNA achieved through loss-of-function mutants of one or all miRNA family members. For example, Arabidopsis plants overexpressing miR159a/b do not display leaf morphogenesis defects [40]. However, miR159a/b double mutants show abnormal leaves that are curled upward [41]. Similarly, overexpressing cleavage resistant targets may not lead to functional characterization of miRNAs, as a miRNA usually regulates multiple target genes with either different or redundant functions. Therefore, expression of all target genes needs to be modulated in order to have insights into miRNA functions. Due to this, functional studies of a 
miRNA family, on most occasions, are possible through simultaneous knockdown of expression of all members of a miRNA family.

Among all available techniques to knockdown miRNAs, an ideal approach is to attract small RNAs by target decoys/mimics, which are partially complementary to the endogenous miRNAs intended to be knocked down. Such technology became possible with the discovery of IPS1 from Arabidopsis, which is the first found endogenous long non-coding RNA decoy that attracts and modulates miR399 activity by a mechanism called Target Mimicry [28]. IPS1 transcripts are partially complementary to miR399 and form a central bulge that blocks miR399mediated cleavage of IPS1. Target mimics function mainly by sequestering the target miRNAs without affecting their abundance [28], which may lead to incomplete inactivation of their activity. Therefore, new approaches that can effectively and specifically inactivate miRNAs were needed in order to study their functions.

Based on the TM approach, a modified technique has recently been developed as the STTM technology, which targets specific endogenous small RNAs for degradation in plants and is effective in knocking down the expression of miRNAs of an entire family [29]. STTM contains two miRNA binding modules, having mismatches at the miRNA cleavage sites, separated by a linker of 48-88 nt that forms a weak stem-loop. STTM has a strong effect in inducing the degradation of the target miRNAs partly through the small degrading nucleases (SDNs) [29]. This technology has been successfully applied to silence numerous miRNA families in Arabidopsis including the multi-membered miR165/166 and miR156/157 families, which could not be efficiently manipulated by IPS1-based TM approach [29, 42]. Furthermore, this technology has been used to knock down miRNAs in major crop plants including tomato [43], rice, tobacco and soybean [44], through stable transgenic plants, agrobacterium-mediated transient expression [45], or virus-mediated approach [46].

Another technology that has been adapted from knocking down miRNA functions in animal cells and applied very recently in plant is the molecular sponges (SPs) [32, 47]. SPs contain 4 copies of miRNA binding sites for effective blockage of miRNAs in animal cells. In contrast, the adapted SP technology for plants contains many more (up to 15) copies of miRNA binding sites with mismatches at the cleavage site to make it effective in knocking down the expression of miRNAs [32, 47]. The higher complementarity of STTMs and SPs than TMs to their target miRNAs may also result in a better binding and silencing efficiency in blocking miRNAs. While SPs use many miRNA binding sites linked by a short (4 nt) untested spacer, STTMs use only tandem miRNA binding motifs in an array, linked by an empirically tested spacer of 48-88 nt that forms a weak "stem-loop" structure to stabilize the overall STTM structures. Two miRNA binding motifs linked by a 4-nt spacer in SPs are similar to the two tandem miRNA binding sites in STTM, linked by a $8 \mathrm{nt}$ spacer, but the structure is not as effective as with a longer spacer, such as 48-88 nt linker, in blocking miRNA activity [29, 47]. This could be due to several reasons (Figure 2). First, short spacer linked with only two miRNA binding motifs could not stabilize SPs and/or STTMs before miRNA associated RNA-induced silencing complexes (miRISCs) could bind to the SPs/STTMs. Second, short spacer possibly leaves limited space for miRISCs binding to the SPS/STTMs, resulting in either a faster turnover of the SPs/STTMs or formation of unstable miRISC-SPs/STTM complexes due to RISC collision. Third, many copies of miRISC binding sites linked by many short spacers may increase the overall stability of SP structure and result in a stronger "sponge" effect in attracting 
miRISCs, though not all the miRNA binding motifs could be occupied by the miRISC due to shorter spacers and RISC collision. This third possibility is an advantage for a "sponge" to bind miRISCs more efficiently through a random collision between the miRNAs and sponge moiety that has multi-miRNA binding sites. In addition, the "central mismatch (cm)" in SPs involves 2nt-mismatches, instead of having a STTM-like "3nt-bulge" at the miRNA cleavage site, which certainly enhances the binding affinity of miRNAs to the miRNA binding sites.

Consequently, TMs, STTMs and SPs have been reported to exhibit variable efficiencies against different miRNA families [47]. For example, TM is not effective to knockdown miR165/166 while STTM and SP are. Similarly, SP and TM have effectively knocked down miR159 while STTM was claimed not to be effective. Such results indicate that the effect of these technologies might vary from one miRNA family to the other or may depend on the interactions between target mimics and different miRNA family members. Such variation may occur due to differences in preference/affinity and or specific efficiencies of different Argonaute (AGO) proteins in sorting miRNAs and their various RNA decoys.

In comparison, construction of up to 15 copies of identical miRNA binding sites in SP is more challenging than making two copies in STTM. Incorporation of advantages of SPs by replacing "3nt-bulge" with a central mismatch (CM) in certain cases, such as miR159, but keeping the single 48-88 nt spacer, may improve the STTM efficiency. Effective STTMs can also be created to target multiple miRNAs at the same time. This objective can be achieved by creating multiplex STTMs, in which individual STTMs can be separated by ribozymes or tRNAs, to target multiple miRNA families in one construct (see discussion in later section). miRNAs whose knockouts give severe or lethal developmental defects, can be characterized using inducible or tissue-specific promoters creating inducible STTMs or tissue-specific STTMs.

Other lesser used techniques to downregulate miRNAs in plants include the use of RNAi [48] and amiRNAs [33]. The RNAi-based approach can be used to silence individual members of a miRNA family. Nevertheless, silencing the entire members of a miRNA family is challenging and the construction of multiple RNAi cassettes in a single vector is hard to achieve due to the limited capacity of a vector. In contrast, amiRNA may overrule that disadvantage of RNAi in silencing miRNAs by giving a flexibility to knockdown the expression of one or all members of a miRNA family using 21-nt amiRNAs [33]. For example, an amiRNA targeting a mature miRNA induces silencing of all miRNA family members, whereas an amiRNA targeting the unique region of a precursor miRNA induces silencing of only the targeted individual family member [33]. So far, limited studies have been reported using amiRNAs to silence endogenous miRNAs in plants. The application of such an approach and comparison with TMs, STTMs and SPs, requires further investigation. Recently, it has been shown that Cas9 fused with the repressor domain and used with gRNAs targeting the promoters of miR159a and $b$, can reduce the expression of both miRNAs [49]. Such an approach can be useful for silencing one to many members of a miRNA family.

\section{amiRNAs and syn-tasiRNAs: Small RNA based silencing of target genes}

amiRNAs are artificially developed or modified miRNAs that mimic endogenous miRNA structures to target and silence any gene of interest. The first generation of gene silencing technology used hairpin RNAi that involves use of sense and antisense gene sequences to 
produce fully complementary double-stranded RNA (dsRNA) as the gene silencing trigger [19, 20]. This was soon paralleled or replaced by the second-generation of RNAi using artificial miRNAs, due to their higher specificity and stability determined by the asymmetric structures of the pre-miRNA secondary structures $[22,50]$. Development of amiRNAs was first suggested by Tang and Galili [51] and shown to be effectively working in plants by groups of Weigel and Eshed [22, 50]. These amiRNAs used various miRNA precursors such as miRNA172a, miR319a, miR164a, miR164b, miR165a, miR165b, miR166g and miR167a in Arabidopsis, and miR528 and miR390 in monocots [52, 53]. These precursors were used as backbones for amiRNA expression, replacing endogenous miRNA/miRNA* with amiRNA/amiRNA* under the control of the constitutive CaMV $35 S$ or other types of promoters.

\section{“One-hit” amiRNAs}

Silencing of a gene by a single amiRNA is termed as "one-hit" amiRNA. miRNAs or amiRNAs are more efficient and have less off-target than traditional RNAi for reasons based on our understanding and knowledge of endogenous miRNA working mechanism. First, most endogenous miRNAs have statistically asymmetrical mismatches along the duplex regions of the miRNA/miRNA*, which allows, in most cases, only one strand, the miRNA, to be recruited by AGO1, while miRNA* strand is excluded/destroyed or sorted to other AGOs [54-56]. Second, conserved miRNAs are structurally and genetically stable in plant genomes due to their longterm evolutionary outcomes [57]. Although amiRNAs that target non-miRNA-regulated genes are artificially designed, their structures are based on the highly conserved miRNA precursors and the secondary structures of the amiRNA duplex regions follow a typical miRNA configuration. Thus, amiRNAs are expected to have a stable gene silencing effect genetically and biochemically.

Unlike animal genes that are often targeted by multiple miRNAs for regulations, plant miRNA-regulated genes are frequently targeted by a single miRNA or a miRNA family composed of multiple members with a highly conserved sequence [58]. Both animal and plant miRNAs are effective and stable in their functions after natural selection during evolution [57]. However, compared to an endogenous miRNA, a newly designed amiRNA may not be as effective or stable in regulating its target gene, due to lack of such an evolutionary process [59]. Different amiRNAs targeting the same gene have shown to have variable effects in knockdown of target gene expression, depending upon the selected target region and/or the precursor miRNA backbones used to express them [22]. To overcome this problem, people often design several amiRNAs to target different regions of the target gene to seek the best amiRNAs for functional analysis of the target gene.

\section{"Two-hit" amiRNAs and syn-tasiRNAs}

Utilization of two amiRNAs to silence a single gene is termed as "two-hit" amiRNA technology. Theoretically, two or more amiRNAs have a higher chance to hit the target gene transcripts for silencing, overcoming the inefficiency of one-hit amiRNA in gene silencing due to the amiRNA targeting positional effect. We have developed a system to clone two or more 
amiRNAs in a single poly-cistronic transcript using the Arabidopsis miR168a backbone. Such two-hit amiRNA approach has been successfully applied to over-express endogenous miR165/166 [60], or to silence SnRK2s [61], in Arabidopsis. In a similar approach, two different amiRNAs were shown to be expressed as one polycistron and found to knockdown their respective target genes [62].

Recently, it has been shown that multiple genes can be targeted by a single construct by using atasiRNA/syn-tasiRNA, where an atasiRNA sequence targeting multiple genes can be constructed using the trans-acting siRNA (TAS) backbone [63]. Such TAS sequence can generate multiple phased atasiRNAs after being triggered by miRNAs, present in the same construct. Similar to the use of different precursor miRNA backbones for amiRNA expression, modulation of syn-tasiRNA expression can be achieved using different TAS loci as a trigger to direct siRNAs production against the target genes. The efficiency of syn-tasiRNAs could be enhanced by multiplexing the syn-tasiRNAs in the TAS sequence to silence a single gene through a single or multiple targeting sites of the gene [64]. This technology makes it possible that multiple genes are silenced by multiple gene-specific syn-tasiRNAs on a single TAS sequence. Production of syn-tasiRNAs requires miRNA triggers such as miR390 and miR173. While miR390 is highly conserved and expressed in plants, not all plants possess miR173. In the latter case, miR173 is often co-expressed with the TAS sequence under either constitutive or inducible promoters to have a regulatory control over gene silencing [34]. Thus, syn-tasiRNAs offer a good alternative for expressing multiplexed artificial small RNAs. A comparison between syn-tasiRNAs and amiRNAs for their efficiencies will be required for further extension and applications of these techniques.

Extension and development of amiRNA technology and its applications in combination with other small RNA-based technologies

As miRNA sequences can be expressed using different types of promoters such as inducible and/or tissue-specific, a temporal and/or spatial repression of the target genes by amiRNAs is possible. The amiRNAs can be cloned in different expression modules, and each module generates a specific amiRNA population from one of the specific miRNA backbones that are linked together by short sequence intervals. A specific miRNA backbone can be modified to have sequence variations to express multiple amiRNAs on a single poly-cistronic amiRNA vector, as used in Arabidopsis to over-express miR165/166 [60], or two $S n R K 2 s$-amiRNAs [61]. Extending two-hits to multiple-hits, up to five amiRNAs in a single polycistron has been successfully used in silencing wheat streak mosaic virus (WSMV) by targeting five different regions of the viral genome [65]. In this case, the five rice miR395 family members in a polycistronic cluster were replaced by five different amiRNAs to target the WSMV. Using this approach, multimeric amiRNAs can be designed to target different genes, or different regions of a single gene for functional genomics. Similar to this, many amiRNAs can be cloned in a construct having multiple modules, to target several genes at the same time. This can be achieved as long as the amiRNA backbones are different or modified differently to prevent structural 
interference in folding their respective stem-loop structures for Dicer-like enzymes (DCLs) to process.

A big challenge in designing amiRNAs is the selection of the target regions to ensure that amiRNAs are highly effective and specific, with less off-target. Screening for effective amiRNAs using epitope-tagged protein-based amiRNA (ETPamir) screens have been developed recently, which involves co-expressing target mRNAs encoding epitope-tagged proteins and the candidate amiRNAs [66, 67]. Alternatively, amiRNAs can be simply evaluated by Agrobacterium-mediated transient expression [68]. Several automated web-based amiRNA design tools such as Weigel's WMD3 (http://wmd3.weigelworld.org) [69] and Carrington's PSAMS (http://p-sams.carringtonlab.org/) [70] have been developed to filter potential off-target small RNAs to ensure highly specific amiRNAs with no off-targets. The amiRNAs with greater specificity, designed through these web-based programs, can be further evaluated by genomewide expression profiling [22] and genome-wide transcriptome profiling combined with 5 RLMRACE [63]. These approaches have helped to design better amiRNAs and test their silencing efficiencies before creating time-consuming stable transgenics. Multiplex amiRNAs targeting one gene can increase gene-silencing efficiency to a greater extent. A simplified procedure for the construction of two-hit or multiplex amiRNAs is necessary for their extension and applications. In case there is a functional redundancy among target genes, we first consider choosing a conserved region of that gene family to knockdown the expression of the entire genes of the family [68]. In cases where functionally redundant family members have no conservations in gene sequences, multiplex amiRNAs can be used in an array to target all family genes.

As discussed previously, amiRNAs can also be applied to silence endogenous miRNA genes [33]. It is generally seen that after knocking down a miRNA by a target mimic, the associated target genes expression is upregulated. In order to simultaneously silence miRNAs and their target genes, amiRNA approach can be combined with STTM technique in one transfer-DNA (T-DNA) construct. Such approach has been successfully used to improve panicle exsertion of rice cytoplasmic male sterile line by fine-tuning the expressions of amiRNA and artificial target mimic [71]. It is possible that such combinatory construct can be expressed under an inducible promoter to control the timing of their expressions for field applications.

\section{CRISPR/Cas9: A guide RNA (gRNA) based potent genome-editing tool for functional genomics}

CRISPR/Cas9 is a recently developed, bacterial-origin, powerful approach to produce targeted mutations in any gene of interest, genome-wide, from prokaryotes to eukaryotes [72-74]. There are two key components that can be manipulated for effective genome editing via CRISPR/Cas9 technology: (1) Cas9 enzyme, a bacterial (Streptococcus pyogenes), RNA-guided DNA endonuclease [75], and (2) Single guide RNA (sgRNA), a synthetic single short RNA made of the fusion of CRISPR RNA (crRNA) and the trans-activating crRNA (tracrRNA) [76]. There are limited studies on the effects of variations in the synthetic sgRNA structures, although the scaffold region of the short sgRNA is easily manipulated [77-79]. On the other hand, studies 
using protein engineering for high-effective Cas9 are also limited, due to the difficulty in handling relatively big sized Cas9 [77]. Furthermore, it is not yet known how much an altered sgRNA scaffold structure affects Cas9 structure and function or vice versa. These aspects require further investigations for an improved CRISPR/Cas9 technology, which are discussed below in detail.

\section{Cas9: A big prokaryotic protein that can be modified for eukaryotic genome editing}

Cas9 is a family of endonuclease enzymes originating from bacteria for defense against foreign DNA invasions. It is about $150 \mathrm{kDa}$, composed of multiple functional domains. It cleaves double-stranded DNA with the help of two interactive small RNAs that can be artificially fused into a single guide RNA (sgRNA) mentioned above [75, 76]. Cas9 enzymes, together with their interactive RNAs or sgRNAs, have recently been crystallized by several groups, which allows us to understand their working mechanisms in a greater detail [77, 80]. Based on their known structures and functions, not all the domains of Cas9 are indispensible for their genome-editing activities. At least REC2 (residues180-307), one of the 20-nt gRNA recognition domains, is not in a direct contact with the bound guide:target heteroduplex, and thus is dispensable [77]. In addition, the protospacer adjacent motif (PAM) interacting (PI) domains of highly related Cas enzymes have variable PAM recognition motifs but are functionally interchangeable as long as their specific PAM recognition motifs are met in gene editing [77].

Recently, Cas9 has been successfully split into parts that can be reconstituted, into a functional unit, through the sgRNA. Split Cas 9 is found to be working as effective as the wild type enzyme, which allows a temporal control of genome editing by an inducible system [78, 79]. Since Cas9 is relatively bigger in size for its manipulation and applications, optimizing the protein structure and making it smaller should be a focus in future studies [81][82]. In fact, efforts towards modulating both Cas9 PAM-specificity and its size minimization have been successful recently, using Cas9 structural information and the bacterial selection-based directed evolution [82]. On the other hand, seeking other types of Cas family proteins, such as the putative class 2 CRISPR effector Cpf1-family proteins, for more effective genome editing has offered new options to improve the CRISPR/Cas system [83].

sgRNA: A key RNA component of Cas9 complex that can be manipulated for high efficient genome editing

The current synthetic sgRNA is composed of a 5' 20-nt protospacer, which recognizes the target gene on a region of 20 bp in a close proximity to the PAM such as NGG, and a 3' scaffold of $76 \mathrm{nt}$ with a defined secondary structure to interact with the endonuclease Cas9 [76]. Although the current sgRNA was created based on the bacterial crRNA and tracrRNA and widely applied in eukaryotes, mutation analysis of sgRNA demonstrated certain flexibility in changes at its various secondary structures [77]. In the scaffold region of the sgRNA, there are sequences composed of, from the 5' to the 3': the so-called repeat, tetra loop, anti-repeat, stemloop 1, linker, stem-loop 2 and stem-loop 3 [77] (Figure 3). Repeat and anti-repeat form a proximal duplex, a distal duplex, and artificial tetra-loop. While mutation of the proximal duplex and deletions of the distal duplex and the linker, mildly affected the Cas9 activities, removal of the bulge between the proximal and distal duplexes and abolishment of stem-loop1 completely knocked out Cas9 activities [77]. These indicate that at least certain variations at the distal 
duplex and linker regions are dispensable in guiding Cas9 to edit the genomic DNA sequences. It can be envisaged that a sgRNA has dispensable and indispensible regions that may or may not induce a change in Cas9 tertiary structure, consequently affecting Cas9 activities and specificities.

\section{Rational design of gRNAs for effective genome editing}

The 20-nt guide RNA (gRNA) is directly linked to the scaffold at the 5' end. Selection of gRNA targeting sites on genes to be edited is also a key step to effectively knock out genes by Cas9. There are several so-called rational designing factors that should be considered for the selection of gRNA targeting sites on a gene to be edited by Cas9 [84]. Among many factors, the protospacer-adjacent motif "PAM" sequences "NGG", recognized by Cas9, has a more preference of " $\mathrm{C}$ " than " $\mathrm{T}$ " for the " $\mathrm{N}$ " base. "G" at position 20 (P20, immediately upstream of "NGG") of the gRNA targeting sites, is highly preferred than other nucleotides, especially "T". Other factors, based on the statistics analysis, are: P9-15 prefer "A"; "C" is not preferred at P3; "G" is not preferred immediately downstream of "NGG". Another independent study showed that gRNAs enriched with guanine and depleted with adenine are more stable and active in gene editing [85]. Furthermore, gRNAs with $5^{\prime}$ mismatches and/or having one or two fewer nucleotides, are as efficient as canonical gRNAs [85]. CRISPRscan, an algorithm that helps in designing highly efficient gRNAs for efficient gene editing in vivo has been developed [85]. In addition, targeting sense and anti-sense strands of the genomic DNA by gRNAs is equally effective in genome editing. Although Cas9 has no preference in recognizing DNA sequences, for a gene knockout by Cas9, it is often most effective to target the upstream region of the open reading frame (ORF). In contrast, 5' and 3' untranslated regions (UTRs) are most ineffective in gene knockout by Cas9. Some of these gRNA-designing guidelines still need further validations [84].

\section{Expression of sgRNA: Constitutive or induciblel tissue-specific}

gRNA, together with the scaffold RNA, is initially expressed under type-III RNA polymerase (pol-III) promoters such as U3/U6 snoRNA promoters in plants and animals. Use of pol-III promoters allows not only the constitutive expression of sgRNA, but also the specific processing of sgRNA at the defined 5' and 3' positions. The latter is essential for the interaction between sgRNA and Cas9, as well as necessary for the activity of Cas9.

For spatial and temporal control of CRISPR/cas9, either sgRNA or Cas9 has to be expressed in spatial and temporal manner. To achieve this, RNA polymerase II (pol II) promoters are to be used instead of pol-III promoters. RNA pol-II promoters are well characterized for their abilities to control gene expressions in a temporal or tissue-specific manner. RNA pol-II promoters, if used, initiate the transcription of RNAs containing a 5' Cap structure together with an undesired 5' sequence before the intended sgRNA. To be active, the sgRNA must be precisely processed at the right position so that the Cas9 can be activated by the processed sgRNA. Along with RNA pol-II promoters, self-cleaving ribozyme (RZ) modules are often fused at both ends of a sgRNA for production of active sgRNAs [86].

Recently, pre-tRNAs were used to fuse with sgRNAs at both the 5' and 3' ends for production of active sgRNAs [87]. In this case, the fused pre-tRNAs-sgRNAs are transcribed by a constitutive pol-III promoter, and are subsequently processed by endogenous tRNA maturation RNases. In this case, the inducible or tissue-specific genome editing can only be achieved by the 
control of Cas9 expression. A series of pre-tRNAs, flanking all gRNAs, in a polycistronic construct can allow multiplexing to target many genes at the same time [87].

Gene mutagenesis by CRISPR/Cas9: Random mutation versus off-target effects

One major concern with CRISPR/Cas9-directed genome editing is its unwanted off-target editing [88-92]. With a sequenced genome, specific and accurate editing to modify genes is important for the application of such technologies. Unlike RNAi, in which off-target effects happen post-transcriptionally, CRISPR/Cas9-directed genome editing is permanent and any offtarget effects will be an unwanted damage to the genome. Studies show that Arabidopsis has a lower off-target effect than that in animal cells [93], but this observation may vary from species to species and requires more studies in different plant systems [92]. Studies indicate the 8-12 nucleotides, of the gRNA, immediately upstream of the "PAM", are crucial in recognizing the gene target sites and are thus termed as the "seed region". While nucleotides outside this seed region are tolerable in genome editing, thus causing off-target effects. Reduction of off-target effects is currently sought by either carefully selecting the target sites through a genome BLAST analysis for gRNA design or reducing the expression of Cas9 [94, 95]. In practice, converting Cas9 activity from a double-stranded DNA (dsDNA) cutter into a single-strand DNA nickase by mutating the RuvC domain of Cas9, dramatically reduces the off-target effects of the wild-type Cas9 [96, 97]. "PAM" structure plays a key role in reducing off-target effects of gRNAs genome-wide and seeking new or engineered Cas9 enzymes with a varied "PAM" interacting (PI) domain will certainly help reduce off-target effects. Recent studies indicate that shortening a 20-nt gRNA to a 17-nt one significantly reduced the off-target effect for specific genes [97, 98]. This indicates that $~ 17-n t$ gRNA had less chance than the 20-nt gRNA in guiding Cas9 to bind to certain off-target positions for undesired editing. However, a shortened gRNA could dramatically increase the genome-wide off-target positions due to its less uniqueness compared with a longer gRNA. Another approach, which significantly reduces off-target effect, is adding two extra target independent guanines at the 5'-terminus of the sgRNA [97]. A recent progress in reducing off-target effect has been achieved through fusing Cas9 protein with any sequencespecific and programmable DNA-binding domain (pDBD). This will help anchor additional DNA sequence that is close to the gRNA-targeting site for extra-accurate DNA recognition and genome editing [99].

On the other hand in plants, frequent gene mutagenesis by CRISPR/Cas9 is desirable in order to expand the mutation spectrum and pool of mutants. In plants, the chemical EMSmediated gene mutagenesis has played a key role in gene identification and functional analysis. The main drawback of EMS-generated mutants is the requirement of a time-consuming mapbased cloning process to identify the mutant gene. In this regard, CRISPR/Cas9 directed gene mutagenesis offers big advantages for faster identification of the mutant genes through sequencing of gRNA genes that are integrated into the transgenic plants. For this matter, the gRNAs can be designed randomly as a mutant pool to generate predictable mutants genomewide. This approach can be more effective by mutations and/or modifications of Cas $9 / \mathrm{sgRNA}$ structures to eliminate the "PAM" requirement. Cas9-directed gene mutagenesis can be 
distinguished from "T-DNA" insertion mutagenesis by segregation of the T-DNA from the gRNA-guided mutation sites [100].

\section{Applications of CRISPR/Cas9 to non-/difficult-transformable plants}

Like other RNA-based technologies, application of CRISPR/Cas9 is dependent on the genetic transformability of the plants. To circumvent genetic transformation, virus-induced gene silencing (VIGS) has been in use for functional genomics [101]. Such an approach may be adapted in facilitating CRISPR-Cas9 to be used in non-/difficult-transformable plants. Virusinduced/mediated genome editing of CRISPR-Cas9 system can reduce tremendous time and labor in generating transgenic plants for each desired mutation. A parent transgenic plant, stably expressing Cas9 protein, can be used to infiltrate gRNAs through viral infection approach. Such infiltrated gRNA can induce desired mutations by reaching the germplasm, which can be carried to subsequent generations. Such approach has recently been successful in tobacco plants [102, 103]. Crop species, which are difficult to transform, can be targeted for genome editing using this approach. The challenge remains that how to pack stably oversized Cas9 gene inside the viral genome or to reduce the Cas9 size for such manipulations. Modification of the viral genome, such as deleting cell-to-cell movement genes in geminivirus to give extra space for Cas9 packing, has been successfully used in tobacco gene editing [104].

\section{Other applications of CRISPR/Cas9 than gene editing in plants}

Finally, CRISPR-Cas9 system can be modified and utilized in functionally activating or repressing any target gene, instead of introducing double stranded DNA breakage. Such can be achieved by converting Cas9 into a non-cleaving DNA binding protein (dCas9) that is further fused with natural/synthetic transcriptional activator and/or repressor such as EDLL/TAL/VP16/VP64 and SRDX/EAR motifs, respectively [49, 105]. Furthermore, CRISPR/Cas9 can mediate sets of scaffold RNAs that can be used to manipulate an entire metabolic pathway governed by many proteins/enzymes and guided by different transcriptional controls (activating or repressing) at various steps [106, 107]. In such cases, some genes can be activated while others can be repressed. A single type of non-cleaving Cas 9 can interact with different modified RNA scaffolds, which provides a potent tool to manipulate the pathways in such a manner enabling regulation of independent gene targets, simultaneously [107]. The minimal CRISPR interference system can silence target genes when dCas9 and sgRNA congregate to block transcription physically [108-110]. To combine both the target and function in a single scaffold RNA, sgRNA molecules recruit RNA-binding proteins that are fused to functional effectors. This is made possible by providing additional aptamer domains to sgRNA, leading to simultaneous activation and repression at the target site [107]. Such a module can be put under temporal control by inducing the dCas9 protein as a master regulator, under an inducible promoter. Such approach when applied to plants can induce potential metabolic changes leading to the engineering of secondary metabolites.

Introducing two gRNAs targeting the same gene can delete the entire region in between those gRNAs and that region can be replaced by a gene of interest in the presence of a homology repair donor [111]. Such approach can be used for activation tagging or tagging genes with reporters such as GFP or luciferase. Thus, homology-directed repair (HDR) of CRISPR/Cas holds great potential for introducing desired gene sequences at desired sites in a plant genome. 
CRISPR-Cas9 system can target not only DNA but also RNA for editing. It has been shown that deoxyribonucleotide-comprised PAMmers (short DNA oligonucleotide having PAM) activated Cas9 to cleave ssRNA in trans [112]. This strategy can be programmed to effectively target only the RNA transcripts but avoiding any cleavage of their corresponding dsDNA template. An orthogonal system can be developed for plants and other organisms to modify desirable mRNA and can prove to be an excellent substitute to RNAi and amiRNA based gene silencing.

\section{Multiplex technology: A potential strategy for functional genomics of gene networks by STTMs, amiRNAs, and sgRNAs}

STTMs, amiRNAs, and sgRNAs are powerful tools in functional genomics of the coding and non-coding genes (Figure 1). The next step is how to use them for functional genomics of gene networks or gene clusters of various pathways. CRISPR/Cas9 sets a good example for such purpose by using multiplex of sgRNAs [37, 87]. Similar to sgRNAs, STTM and amiRNAs are also relatively small in size with certain stable secondary structures, and thus can be hypothetically multiplexed. Though, their stabilities upon multiplexing require further investigations. There are a few ways with different advantages and disadvantages for such multiplex approaches as discussed below.

\section{Multiplex by multiple transcriptional units}

As the initial multiplex of sgRNAs for CRISPR/Cas9, a transcriptional unit of sgRNA using pol-III promoter and terminator can be repeatedly used in a vector to express multiple sgRNAs for editing multiple genes at a time [49, 113]. Such a strategy can be used for STTMs and amiRNAs with the difference that STTMs and amiRNAs normally use a pol-II promoter and a longer terminator, instead of pol-III transcriptional unit, which has a shorter pol-III promoter and "TTTTT" as terminator. The oversized pol-II transcriptional unit limits the repeated use of such system for multiplex of STTMs and amiRNAs. Nevertheless, STTMs and amiRNAs can be multiplexed using a single long poly-cistronic transcript having multiple STTM or amiRNA structures. The disadvantage of poly-cistronic transcript is that STTMs have identical spacers between miRNA binding domains and repeated use of such spacer may lead to the misfolding of the multiplexed STTMs. This can interfere with their binding to the targeted miRNAs. Similar situations may also be true to the multiplex of amiRNAs using a single long poly-cistronic transcript that have misfolded pre-amiRNA structures. In such cases, other strategies such as self-cleaving ribozyme-mediated or tRNA-mediated multiplex of STTMs or amiRNAs may be used (Figure 4) [86, 87].

\section{Multiplex by multiple self-cleaving ribozyme structures on a single transcript}

Self-cleaving ribozyme structures have great potential to mediate the multiplex of sgRNAs as shown for a single sgRNA processing in budding yeast and in vitro, and for multiple sgRNAs in Leishmania [86, 114]. However, this system still remains to be tested in plants. Hammerhead $(\mathrm{HH})$ and hepatitis delta virus (HDV) ribozymes (RZ) are well studied and frequently used for such purposes (Figure 4). There are several advantages of using ribozymes. First, utilization of promoter system has no limitations, which can be either pol-II or pol-III. Second, each individual sgRNA, STTM, or amiRNA can be physically separated without interfering with each other. Finally, the regeneration of small RNA expression units for the 
multiplex can be simply mediated by using type-IIS endonucleases, such as BsaI and BbsI, with the help of a unique antibiotics selection marker between the two BsaI sites [113].

\section{Multiplex by multiple pre-tRNA structures on a single transcript}

Self-cleaving ribozymes mediate the cleavages of sgRNA at specific sites of the 5' and 3' ends. Pre-tRNA transcripts are processed by the constitutively expressed endogenous enzymes called RNases P and Z (Figure 4B) [87]. Since tRNA biogenesis is so conserved among eukaryotes, properly engineering pre-tRNAs at the 5' and 3' ends of the sgRNAs can generate precise cleavage of sgRNAs by the highly conserved endogenous RNases (Figure 4). The two internal transcriptional elements, termed Boxes A and B, inside the tRNA could collaborate with pol-III promoters to boost the expression of pre-tRNA-sgRNA-pre-tRNA (TGT) or polycistronic pre-tRNA-sgRNA (PTG) transcripts to produce one or numerous sgRNAs for a multiplex purpose [87]. Furthermore, chromosomal-fragment deletion can be achieved using the minimal multiplex (two gRNAs). Such strategy was successful in targeting a single gene for a segment deletion at a high efficiency. This will eventually cause the entire region of selection to be deleted between the two gRNA-binding sites. Like "two-hit" amiRNAs, using two or more gRNAs for editing a single gene not only increases the efficiency of the gene mutation but also increases the chances that a knockout allele is created in the process. This approach could be useful for long-range genome editing to eliminate unnecessary genome sequences in application of optimizing a mini-chromosome and its behavior in cells [115]. Considering the conserved nature of tRNA based processing across all organisms, pre-tRNA based gRNA design has currently been used in plants and can be universally applied to all organisms. [87].

Ribozymes and tRNA-based multiplexing holds great potential, yet to be tested for multiplexing STTMs and amiRNAs in plants. One concern over multiplexing STTMs and amiRNAs is their potential instability in the absence of cap and polyA tail after being cleaved by ribozymes or tRNA-specific endonuclease. This is not the case with multiplexing sgRNAs, where their stability is not affected in the absence of cap and polyA tail.

\section{Concluding remarks and future perspectives}

RNA based approaches such as the highlighted STTM, amiRNA and gRNA/Cas9 hold huge potential to study gene functions by knocking-down, knocking-out, knocking-in and upregulating gene expressions. STTMs and amiRNAs are extremely useful in functional study of small RNAs and coding genes, respectively. While application of STTMs and amiRNAs largely relies on transgenic technology to integrate them into genomes for function, Cas9 and its associated sgRNA are only temporarily required for inducing DNA breakage for mutation at specific locations in the genome. Once mutated, Cas9-sgRNA, existing as a T-DNA, can be segregated out via genetic crossing [100]. To eliminate the problem of creating transgenic plants carrying T-DNA, protoplasts from various species, transfected with sgRNA-Cas9 protein complexes, have been successfully regenerated into plants with specific gene edited [116]. This provides a great potential for crop improvement without any concern of transgenic issues. Nevertheless, plant regeneration from protoplasts will become an essential means for this application. Cas9 based technology is especially useful in fast generation of all kinds of mutants for functional genomics in plants and even in applications in generating resources for crop breeding. Furthermore, Cas9 technology can be extended for transcriptional activation, suppression, or tight regulation of genes by fusing it with various transcriptional activators 
and/or suppressors. Such transcriptional regulation of genes can also be achieved through the engineering of the sgRNAs at the 3' end. These flexible applications, together with the use of constitutive, inducible or tissue-specific expressional promoters, are able to dissect any desired genes or gene networks for their functions.

Future directions for application of these technologies will still be focused on efficiency, accuracy, simplicity, and reliability. These could be achieved by further optimizing the RNA structures of STTMs, amiRNAs, and sgRNAs. In this regard, we shall investigate the following but not limited to: (1) the best dynamic interacting structures between miRNAs and miRNA target mimics for inactivating or triggering degradation of miRNAs; (2) the functions of endogenous miRNA backbones and their modifications for the design of amiRNAs; and (3) the best structure of sgRNA scaffold for genome editing by mutation and modification, such as introducing a flip-AU at the scaffold region [117]. Certainly, these RNA-protein interacting components can be optimized not only through the change of the RNA partners, but also through their biogenesis pathway components by engineering a better protein/enzyme partner such as SDNs, DCLs/AGOs, or Cas9. Split Cas9 is one of such examples in optimization for better genome editing $[78,79]$.

\section{Acknowledgements}

This study was partially supported by funds from Henan Agricultural University (HAU) and NSFC (No. 31571679), China. G.T. is supported by NSF grants (IOS-1048216 and IOS-

1340001). S. T. is supported by a post-doctoral scholarship from HAU. X. T. is supported by National Institutes of Health grants NIH K01 DK078648, R03 DK084166 and 1R15DK10319701A1. We are thankful to anonymous reviewers for their critical and constructive suggestions to improve this article. We are also thankful to Michigan Tech Multiliteracies Center (MTMC) for improving English of the article. 


\section{References}

1 Peters, J.L., et al. (2003) Forward genetics and map-based cloning approaches. Trends in plant science 8, 484-491

2 Rosso, M.G., et al. (2003) An Arabidopsis thaliana T-DNA mutagenized population (GABIKat) for flanking sequence tag-based reverse genetics. Plant molecular biology 53, 247-259

3 Parinov, S. and Sundaresan, V. (2000) Functional genomics in Arabidopsis: large-scale insertional mutagenesis complements the genome sequencing project. Current opinion in biotechnology 11, 157-161

4 Morozova, O. and Marra, M.A. (2008) Applications of next-generation sequencing technologies in functional genomics. Genomics 92, 255-264

5 Kuromori, T., et al. (2009) Phenome analysis in plant species using loss-of-function and gainof-function mutants. Plant \& cell physiology 50, 1215-1231

6 Weigel, D., et al. (2000) Activation tagging in Arabidopsis. Plant Physiol 122, 1003-1013

7 Kim, Y., et al. (2006) EMS mutagenesis of Arabidopsis. Methods in molecular biology 323, 101-103

8 Weigel, D. and Glazebrook, J. (2006) EMS Mutagenesis of Arabidopsis Seed. CSH protocols 2006

9 Azpiroz-Leehan, R. and Feldmann, K.A. (1997) T-DNA insertion mutagenesis in Arabidopsis: going back and forth. Trends in genetics : TIG 13, 152-156

10 Walbot, V. (1992) Strategies for Mutagenesis and Gene Cloning Using Transposon Tagging and T-DNA Insertional Mutagenesis. Annual Review of Plant Physiology and Plant Molecular Biology 43, 49-82

11 Urnov, F.D., et al. (2010) Genome editing with engineered zinc finger nucleases. Nat Rev Genet 11, 636-646

12 Zhang, Y., et al. (2013) Transcription Activator-Like Effector Nucleases Enable Efficient Plant Genome Engineering. Plant physiology 161, 20-27

13 Marciada, M.J., et al. (2008) Crystal structure of I-Dmol in complex with its target DNA provides new insights into meganuclease engineering. P Natl Acad Sci USA 105, 1688816893

14 Shan, Q.W., et al. (2013) Targeted genome modification of crop plants using a CRISPR-Cas system. Nat Biotechnol 31, 686-688

15 Xie, K.B. and Yang, Y.N. (2013) RNA-Guided Genome Editing in Plants Using a CRISPRCas System. Mol Plant 6, 1975-1983

16 Hamilton, A.J. and Baulcombe, D.C. (1999) A species of small antisense RNA in posttranscriptional gene silencing in plants. Science 286, 950-952

17 Baulcombe, D. (2004) RNA silencing in plants. Nature 431, 356-363 
18 Fire, A., et al. (1998) Potent and specific genetic interference by double-stranded RNA in Caenorhabditis elegans. Nature 391, 806-811

19 Smith, N.A., et al. (2000) Gene expression - Total silencing by intron-spliced hairpin RNAs. Nature 407, 319-320

20 Chuang, C.F. and Meyerowitz, E.M. (2000) Specific and heritable genetic interference by double-stranded RNA in Arabidopsis thaliana. P Natl Acad Sci USA 97, 4985-4990

21 Wesley, S.V., et al. (2003) Custom knock-outs with hairpin RNA-mediated gene silencing. Methods in molecular biology 236, 273-286

22 Schwab, R., et al. (2006) Highly specific gene silencing by artificial microRNAs in Arabidopsis. Plant Cell 18, 1121-1133

23 Morel, J.B., et al. (2000) DNA methylation and chromatin structure affect transcriptional and post-transcriptional transgene silencing in Arabidopsis. Curr Biol 10, 1591-1594

24 Sijen, T., et al. (2001) Transcriptional and posttranscriptional gene silencing are mechanistically related. Curr Biol 11, 436-440

25 Vaucheret, H. and Fagard, M. (2001) Transcriptional gene silencing in plants: targets, inducers and regulators. Trends in Genetics 17, 29-35

26 Tang, G.L. (2005) siRNA and miRNA: an insight into RISCs. Trends Biochem Sci 30, 106114

27 Kozomara, A. and Griffiths-Jones, S. (2011) miRBase: integrating microRNA annotation and deep-sequencing data. Nucleic Acids Res 39, D152-D157

28 Franco-Zorrilla, J.M., et al. (2007) Target mimicry provides a new mechanism for regulation of microRNA activity. Nat Genet 39, 1033-1037

29 Yan, J., et al. (2012) Effective Small RNA Destruction by the Expression of a Short Tandem Target Mimic in Arabidopsis. Plant Cell 24, 415-427

30 Tang, G.L. and Tang, X.Q. (2013) Short Tandem Target Mimic: A Long Journey to the Engineered Molecular Landmine for Selective Destruction/Blockage of MicroRNAs in Plants and Animals. J Genet Genomics 40, 291-296

31 Tang, G.L., et al. (2012) Construction of short tandem target mimic (STTM) to block the functions of plant and animal microRNAs. Methods 58, 118-125

32 Ebert, M.S., et al. (2007) MicroRNA sponges: competitive inhibitors of small RNAs in mammalian cells. Nat Methods 4, 721-726

33 Eamens, A.L., et al. (2011) Efficient silencing of endogenous microRNAs using artificial microRNAs in Arabidopsis thaliana. Mol Plant 4, 157-170

34 Zhang, Z.J. (2014) Artificial trans-acting small interfering RNA: a tool for plant biology study and crop improvements. Planta 239, 1139-1146

35 Jiang, Q., et al. (2014) Small indels induced by CRISPR/Cas9 in the 5 ' region of microRNA lead to its depletion and Drosha processing retardance. Rna Biol 11, 1243-1249

36 Zhao, Y.C., et al. (2014) Sequence-specific inhibition of microRNA via CRISPR/CRISPRi system. Sci Rep-Uk 4

37 Cong, L., et al. (2013) Multiplex genome engineering using CRISPR/Cas systems. Science $339,819-823$

38 Tang, G.L. (2010) Plant microRNAs: An insight into their gene structures and evolution. Semin Cell Dev Biol 21, 782-789

39 Jiang, D.H., et al. (2006) Duplication and expression analysis of multicopy miRNA gene family members in Arabidopsis and rice. Cell Res 16, 507-518 
40 Palatnik, J.F., et al. (2007) Sequence and expression differences underlie functional specialization of Arabidopsis microRNAs miR159 and miR319. Dev Cell 13, 115-125

41 Allen, R.S., et al. (2007) Genetic analysis reveals functional redundancy and the major target genes of the Arabidopsis miR159 family. Proc Natl Acad Sci U S A 104, 16371-16376

42 Todesco, M., et al. (2010) A Collection of Target Mimics for Comprehensive Analysis of MicroRNA Function in Arabidopsis thaliana. Plos Genet 6

43 Jia, X.Y., et al. (2015) Functional plasticity of miR165/166 in plant development revealed by small tandem target mimic. Plant Sci 233, 11-21

44 Wong, J., et al. (2014) Roles of small RNAs in soybean defense against Phytophthora sojae infection. Plant J 79, 928-940

45 Tang, Y., et al. (2013) Virus-induced gene silencing using artificial miRNAs in Nicotiana benthamiana. Methods in molecular biology 975, 99-107

46 Sha, A.H., et al. (2014) Virus-Based MicroRNA Silencing in Plants. Plant physiology 164, 36-47

47 Reichel, M., et al. (2015) Inhibiting plant microRNA activity: molecular SPONGEs, target MIMICs and STTMs all display variable efficacies against target microRNAs. Plant biotechnology journal

48 Vaistij, F.E., et al. (2010) Suppression of microRNA accumulation via RNA interference in Arabidopsis thaliana. Plant Mol Biol 73, 391-397

49 Lowder, L.G., et al. (2015) A CRISPR/Cas9 Toolbox for Multiplexed Plant Genome Editing and Transcriptional Regulation. Plant Physiol 169, 971-985

50 Alvarez, J.P., et al. (2006) Endogenous and synthetic microRNAs stimulate simultaneous, efficient, and localized regulation of multiple targets in diverse species. Plant Cell 18, 11341151

51 Tang, G.L. and Galili, G. (2004) Using RNAi to improve plant nutritional value: from mechanism to application. Trends Biotechnol 22, 463-469

52 Warthmann, N., et al. (2008) Highly specific gene silencing by artificial miRNAs in rice. PLoS One 3, e1829

53 Carbonell, A., et al. (2015) Highly specific gene silencing in a monocot species by artificial microRNAs derived from chimeric miRNA precursors. Plant J 82, 1061-1075

54 Khvorova, A., et al. (2003) Functional siRNAs and rniRNAs exhibit strand bias. Cell 115, 209-216

55 Schwarz, D.S., et al. (2003) Asymmetry in the assembly of the RNAi enzyme complex. Cell $115,199-208$

56 Okamura, K., et al. (2009) Distinct Mechanisms for MicroRNA Strand Selection by Drosophila Argonautes. Mol Cell 36, 431-444

57 Tang, G. (2010) Plant microRNAs: an insight into their gene structures and evolution. Semin Cell Dev Biol 21, 782-789

58 Tang, G., et al. (2008) The art of microRNA: various strategies leading to gene silencing via an ancient pathway. Biochim Biophys Acta 1779, 655-662

59 Takuno, S. and Innan, H. (2008) Evolution of complexity in miRNA-mediated gene regulation systems. Trends Genet 24, 56-59

$60 \mathrm{Ji}, \mathrm{L}$., et al. (2011) ARGONAUTE10 and ARGONAUTE1 regulate the termination of floral stem cells through two microRNAs in Arabidopsis. PLoS Genet 7, e1001358

61 Cai, Z.Y., et al. (2014) GSK3-like kinases positively modulate abscisic acid signaling through phosphorylating subgroup III SnRK2s in Arabidopsis. P Natl Acad Sci USA 111, 9651-9656 
62 Liang, G., et al. (2012) A new strategy for construction of artificial miRNA vectors in Arabidopsis. Planta 235, 1421-1429

63 Carbonell, A., et al. (2014) New generation of artificial MicroRNA and synthetic trans-acting small interfering RNA vectors for efficient gene silencing in Arabidopsis. Plant Physiol 165, 15-29

64 de la Luz Gutierrez-Nava, M., et al. (2008) Artificial trans-acting siRNAs confer consistent and effective gene silencing. Plant Physiol 147, 543-551

65 Fahim, M., et al. (2012) Resistance to Wheat streak mosaic virus generated by expression of an artificial polycistronic microRNA in wheat. Plant Biotechnol J 10, 150-163

66 Li, J.F., et al. (2013) Comprehensive protein-based artificial microRNA screens for effective gene silencing in plants. Plant Cell 25, 1507-1522

$67 \mathrm{Li}$, J.F., et al. (2014) Epitope-tagged protein-based artificial miRNA screens for optimized gene silencing in plants. Nature protocols 9, 939-949

68 Bhagwat, B., et al. (2013) An in vivo Transient Expression System Can Be Applied for Rapid and Effective Selection of Artificial MicroRNA Constructs for Plant Stable Genetic Transformation. J Genet Genomics 40, 261-270

69 Ossowski, S., et al. (2008) Gene silencing in plants using artificial microRNAs and other small RNAs. Plant J 53, 674-690

70 Fahlgren, N., et al. (2015) P-SAMS: a web site for plant artificial microRNA and synthetic trans-acting small interfering RNA design. Bioinformatics

71 Chen, H., et al. (2013) Improving panicle exsertion of rice cytoplasmic male sterile line by combination of artificial microRNA and artificial target mimic. Plant Biotechnol J 11, 336343

72 Sternberg, S.H. and Doudna, J.A. (2015) Expanding the Biologist's Toolkit with CRISPRCas9. Mol Cell 58, 568-574

73 Shalem, O., et al. (2015) High-throughput functional genomics using CRISPR-Cas9. Nat Rev Genet 16, 299-311

74 Ishino, Y., et al. (1987) Nucleotide-Sequence of the Iap Gene, Responsible for AlkalinePhosphatase Isozyme Conversion in Escherichia-Coli, and Identification of the GeneProduct. J Bacteriol 169, 5429-5433

75 Deltcheva, E., et al. (2011) CRISPR RNA maturation by trans-encoded small RNA and host factor RNase III. Nature 471, 602-607

76 Jinek, M., et al. (2012) A programmable dual-RNA-guided DNA endonuclease in adaptive bacterial immunity. Science 337, 816-821

77 Nishimasu, H., et al. (2014) Crystal structure of Cas9 in complex with guide RNA and target DNA. Cell 156, 935-949

78 Wright, A.V., et al. (2015) Rational design of a split-Cas9 enzyme complex. Proc Natl Acad Sci U S A 112, 2984-2989

79 Zetsche, B., et al. (2015) A split-Cas9 architecture for inducible genome editing and transcription modulation. Nat Biotechnol 33, 139-142

80 Jinek, M., et al. (2014) Structures of Cas9 endonucleases reveal RNA-mediated conformational activation. Science 343, 1247997

81 Ran, F.A., et al. (2015) In vivo genome editing using Staphylococcus aureus Cas9. Nature 520, 186-191

82 Kleinstiver, B.P., et al. (2015) Engineered CRISPR-Cas9 nucleases with altered PAM specificities. Nature 523, 481-485 
83 Zetsche, B., et al. (2015) Cpf1 Is a Single RNA-Guided Endonuclease of a Class 2 CRISPRCas System. Cell 163, 759-771

84 Doench, J.G., et al. (2014) Rational design of highly active sgRNAs for CRISPR-Cas9mediated gene inactivation. Nat Biotechnol 32, 1262-1267

85 Moreno-Mateos, M.A., et al. (2015) CRISPRscan: designing highly efficient sgRNAs for CRISPR-Cas9 targeting in vivo. Nat Methods 12, 982-988

86 Gao, Y. and Zhao, Y. (2014) Self-processing of ribozyme-flanked RNAs into guide RNAs in vitro and in vivo for CRISPR-mediated genome editing. J Integr Plant Biol 56, 343-349

87 Xie, K., et al. (2015) Boosting CRISPR/Cas9 multiplex editing capability with the endogenous tRNA-processing system. Proc Natl Acad Sci U S A 112, 3570-3575

88 Cradick, T.J., et al. (2013) CRISPR/Cas9 systems targeting beta-globin and CCR5 genes have substantial off-target activity. Nucleic Acids Res 41, 9584-9592

89 Hruscha, A., et al. (2013) Efficient CRISPR/Cas9 genome editing with low off-target effects in zebrafish. Development 140, 4982-4987

90 Pattanayak, V., et al. (2013) High-throughput profiling of off-target DNA cleavage reveals RNA-programmed Cas9 nuclease specificity. Nat Biotechnol 31, 839-843

$91 \mathrm{Kuscu}, \mathrm{C}$., et al. (2014) Genome-wide analysis reveals characteristics of off-target sites bound by the Cas9 endonuclease. Nat Biotechnol 32, 677-683

92 Endo, M., et al. (2015) Multigene knockout utilizing off-target mutations of the CRISPR/Cas9 system in rice. Plant \& cell physiology 56, 41-47

93 Feng, Z., et al. (2014) Multigeneration analysis reveals the inheritance, specificity, and patterns of CRISPR/Cas-induced gene modifications in Arabidopsis. Proc Natl Acad Sci U S A $111,4632-4637$

94 Bae, S., et al. (2014) Cas-OFFinder: a fast and versatile algorithm that searches for potential off-target sites of Cas9 RNA-guided endonucleases. Bioinformatics 30, 1473-1475

95 Xiao, A., et al. (2014) CasOT: a genome-wide Cas9/gRNA off-target searching tool. Bioinformatics

96 Shen, B., et al. (2014) Efficient genome modification by CRISPR-Cas9 nickase with minimal off-target effects. Nat Methods 11, 399-402

97 Cho, S.W., et al. (2014) Analysis of off-target effects of CRISPR/Cas-derived RNA-guided endonucleases and nickases. Genome research 24, 132-141

$98 \mathrm{Fu}$, Y., et al. (2014) Improving CRISPR-Cas nuclease specificity using truncated guide RNAs. Nat Biotechnol 32, 279-284

99 Bolukbasi, M.F., et al. (2015) DNA-binding-domain fusions enhance the targeting range and precision of Cas9. Nat Methods

$100 \mathrm{Xu}$, R.F., et al. (2015) Generation of inheritable and "transgene clean" targeted genomemodified rice in later generations using the CRISPR/Cas9 system. Scientific reports 5, 11491

101 Becker, A. and Lange, M. (2010) VIGS--genomics goes functional. Trends in plant science $15,1-4$

102 Ali, Z., et al. (2015) Efficient Virus-Mediated Genome Editing in Plants Using the CRISPR/Cas9 System. Mol Plant 8, 1288-1291

103 Yin, K., et al. (2015) A geminivirus-based guide RNA delivery system for CRISPR/Cas9 mediated plant genome editing. Sci Rep 5, 14926

104 Baltes, N.J., et al. (2014) DNA replicons for plant genome engineering. Plant Cell 26, 151163 
105 Piatek, A., et al. (2015) RNA-guided transcriptional regulation in planta via synthetic dCas9-based transcription factors. Plant Biotechnol J 13, 578-589

106 Konermann, S., et al. (2015) Genome-scale transcriptional activation by an engineered CRISPR-Cas9 complex. Nature 517, 583-588

107 Zalatan, J.G., et al. (2015) Engineering complex synthetic transcriptional programs with CRISPR RNA scaffolds. Cell 160, 339-350

108 Qi, L.S., et al. (2013) Repurposing CRISPR as an RNA-guided platform for sequencespecific control of gene expression. Cell 152, 1173-1183

109 Larson, M.H., et al. (2013) CRISPR interference (CRISPRi) for sequence-specific control of gene expression. Nature protocols 8, 2180-2196

110 Gilbert, L.A., et al. (2013) CRISPR-mediated modular RNA-guided regulation of transcription in eukaryotes. Cell 154, 442-451

111 Zheng, Q., et al. (2014) Precise gene deletion and replacement using the CRISPR/Cas9 system in human cells. Biotechniques 57, 115-124

112 O'Connell, M.R., et al. (2014) Programmable RNA recognition and cleavage by CRISPR/Cas9. Nature 516, 263-266

113 Xing, H.L., et al. (2014) A CRISPR/Cas9 toolkit for multiplex genome editing in plants. BMC plant biology 14, 327

114 Zhang, W.W. and Matlashewski, G. (2015) CRISPR-Cas9-Mediated Genome Editing in Leishmania donovani. mBio 6, e00861

115 Han, F., et al. (2007) Minichromosome analysis of chromosome pairing, disjunction, and sister chromatid cohesion in maize. Plant Cell 19, 3853-3863

116 Woo, J.W., et al. (2015) DNA-free genome editing in plants with preassembled CRISPRCas9 ribonucleoproteins. Nat Biotechnol

117 Chen, B., et al. (2013) Dynamic imaging of genomic loci in living human cells by an optimized CRISPR/Cas system. Cell 155, 1479-1491

\section{Glossary:}

Artificial miRNA: Artificial microRNA (amiRNA) is a customized 21-mer small RNA artificially made by using a modified backbone of an endogenous precursor miRNA (premiRNA), which is generated through the endogenous miRNA pathway. The amiRNA can be designed and generated to target any gene or gene family of interest for effective silencing.

atasiRNA/syn-tasiRNA: Artificial/synthetic trans-active siRNA (atasiRNA/syn-tasiRNA) is a customized 21-mer small RNA made artificially by using a modified backbone of an endogenous trans-active siRNA (tasiRNA) precursor, such as TAS1 and TAS3, from tasiRNA pathway. The atasiRNA/syn-tasiRNA can be designed and generated to target any gene or gene family of interest for silencing.

Cas9: CRISPR associated protein 9 (Cas9) is a DNA endonuclease which is utilized to edit genomes with the help of guide RNA. It requires two interacting small RNAs termed CRISPR RNA (crRNA) and trans-activating crRNA (tracrRNA) for function in bacteria. Cas9 is now modified to function in eukaryotic cells for wide applications including genome editing for gene 
mutation and modification. Cas9 is also modified to regulate gene expressions through transcriptional activation and suppression.

CRISPR: Clustered regularly interspaced short palindromic repeats (CRISPR) is referred to as short, palindromic repeats of DNA sequences found in the genomes of some microorganisms, acquired previously in response to a bacterial virus or plasmid invasion. CRISPR is transcribed into pre-crRNA and tracrRNA that hybridize with each other to form a partial double-stranded RNA complex to be processed by bacterial RNaseIII into mature crRNA/tracrRNA complex. This mature crRNA/tracrRNA complex is recruited by Cas9 enzyme to recognize and cleave the DNA, through the 5' section of the crRNA termed guide RNA (gRNA).

Single guide RNA: The single guide RNA (sgRNA) is a chimera of the crRNA and tracrRNA, which combines the targeting specificity of the former and the scaffolding properties of the latter. sgRNA is required in directing Cas9 protein to bind and edit the target gene at a position upstream of the protospacer adjacent motif (PAM) through base-pairing.

MicroRNA sponge: miRNA sponge (SP) is an artificial RNA containing many copies (4-15) of miRNA binding sites that can bind to target miRNAs without being cleaved by them. miRNA SP serves as a decoy for a miRNA by sequestering miRNA away from their endogenous targets, derepressing the target gene expressions.

Multiplex: It refers to a way to express multiple artificial genes by either a single transcription unit or multiple transcription units, sometimes with the help of post-transcriptional modification through ribozyme (RZ) or pre-tRNA machinery.

Ribozyme: Ribozyme (RZ) is an RNA in structure but functions as an enzyme, such as the selfcleavage Hammerhead (HH) and hepatitis delta virus (HDV) ribozymes mentioned in this article.

tRNA: Transfer RNAs (tRNAs) are 75 to 95 nucleotides long RNA molecules which act as carriers of amino acids during protein synthesis. Mature tRNA is derived from pre-tRNA through the processing of RNase $\mathrm{P}$ and $\mathrm{Z}$.

STTM: Short tandem target mimic (STTM) is an artificial RNA structure having two miRNA binding sites that are linked by an RNA spacer of 48-88 nucleotides (nt). STTM can bind to target miRNAs without being cleaved by them. STTMs inactivate miRNA activity by degrading and/or sequestering miRNA away from its target gene transcripts.

Target mimicry: Target mimicry (TM) is a phenomenon when a non-coding RNA, having a non-cleavable miRNA-binding site, mimics and competes with the miRNA target RNA to bind to a miRNA. This, in turn, offers a protection for the miRNA target by sequestering the miRNA away from the target. This phenomenon was first observed in Arabidopsis that the non-coding gene INDUCED BY PHOSPHATE STARVATION1 (IPS1) binds and sequesters miR399 from its target, $\mathrm{PHO}$, a putative ubiquitin-conjugating enzyme E2 24. TM serves as a basis for the recently developed STTM and SP technology and both can be engineered to target and block the functions of any miRNA of interest. 


\section{Figure Legends}

Figure 1. A few essential RNA-based technologies to address gene functions (A) STTM technology for characterizing functions of non-coding miRNA genes. (B) Artificial miRNA (amiRNA) technology for functions of coding genes. (C) CRISPR/Cas9 technology for gene editing.

Figure 2. Comparison of STTM technology with target mimic (TM) and Sponge (SP) technologies. (A) Endogenous IPS1 backbone-based TM technology showing the bulge (NNN), wobble (:) and mismatch (X) between miRNA and the miRNA binding site on the non-coding IPS1 RNA [28]. (B) The improved STTM technology based on TM, showing a stronger interaction between the two miRNAs and the two miRNA binding sites that are linked by a 48$88 \mathrm{nt}$ spacer. The complementary regions show only bulges (NNN) with no wobbles/mismatches [29]. (C) The SP technology shows many copies (4-15) of miRNA binding sites with central mismatches (XX), which are linked by many (3-14) 4-nt spacers between miRNA binding sites [47].

Figure 3. The secondary structure of a sgRNA complexed with the target DNA and its detailed domain structures. (A) The crystal structure of the sgRNA published by Nishimasu et al [77]. Structure was made by the Chimera program. The 20 nucleotides of the genomic DNA target site were specified with 1.C-20.C in yellow. The 20 nucleotides of the gRNA section on the sgRNA were specified with 1.B-20.B in tan. The repeat and anti-repeat are in red and blue, respectively. The repeat and anti-repeat form proximal and distal duplexes as well as a bulge between them, which are indicated with dashed circles and their names. Tetra-loop is in rosy brown. Stem-loop 1, 2 and 3 are in cyan, orange, and purple, respectively. The linker between stem-loop 1 and 2 is in green. (B) The genomic DNA site that is targeted by sgRNA and the DNA nucleotides are specified in letter $\mathrm{C}$ corresponding to the indicated crystal structure made by the Chimera program. NGG is indicated on the complementary strand of the gRNA target site. (C) The sgRNA sequence in detail. The domain names, letter symbols and nucleotides are corresponding to the sgRNA crystal structure with the same color codes. Letter "I" indicates base pairing.

Figure 4. Multiplex for application of RNA-based technologies. Multiplex is usually accomplished by repeated use of the transcriptional units such as RNA polymerase III (Pol-III)based transcription of sgRNAs for expressing multiple gRNAs to edit multiple genes (A) in CRISPR/Cas9. This technology can also be fulfilled by using a single transcriptional unit that contains multiple sgRNAs, linked by multiple pre-tRNAs (B) [87], or ribozymes such as hammerhead $(\mathrm{HH})$ and hepatitis delta virus (HDV) ribozyme sequences $(\mathrm{C})[86,114]$. 
Table 1. Design of RNA-based technologies, their working mechanisms, advantages and disadvantages

\begin{tabular}{|c|c|c|c|c|}
\hline $\begin{array}{l}\text { Name of the } \\
\text { Technology }\end{array}$ & Design & $\begin{array}{c}\text { Working } \\
\text { Mechanism }\end{array}$ & Advantages & Disadvantages \\
\hline $\begin{array}{l}\text { Artificial } \\
\text { miRNA }\end{array}$ & $\begin{array}{l}\text { An artificially designed 21- } \\
\text { nt miRNA that can be } \\
\text { expressed using an existing } \\
\text { miRNA precursor backbone } \\
\text { in cells to target the RNA } \\
\text { transcripts of any gene of } \\
\text { interest for silencing. }\end{array}$ & $\begin{array}{l}\text { RISC-RNA } \\
\text { interaction } \\
\text { (requires the } \\
\text { miRNA } \\
\text { pathway) }\end{array}$ & $\begin{array}{l}\text { More effective in } \\
\text { knockdown of } \\
\text { target gene } \\
\text { expression and } \\
\text { have fewer off- } \\
\text { target effects than } \\
\text { traditional RNAi. } \\
\text { Able to silence } \\
\text { both coding and } \\
\text { non-coding genes } \\
\text { such as single or } \\
\text { multiple } \\
\text { members of a } \\
\text { miRNA family. }\end{array}$ & $\begin{array}{l}\text { It is a knockdown } \\
\text { at RNA and } \\
\text { protein levels } \\
\text { rather than a } \\
\text { knockout at the } \\
\text { DNA level. }\end{array}$ \\
\hline $\begin{array}{c}\text { atasiRNAs/ } \\
\text { syn-tasiRNAs }\end{array}$ & $\begin{array}{l}\text { A customized } 21 \text {-mer small } \\
\text { interfering RNA (siRNA) } \\
\text { made artificially by using a } \\
\text { (modified) backbone of an } \\
\text { endogenous trans-active } \\
\text { siRNA (tasiRNA) precursor, } \\
\text { such as TAS1 and TAS3, } \\
\text { that can be designed and } \\
\text { generated to target any gene } \\
\text { or gene family of interest for } \\
\text { silencing. }\end{array}$ & $\begin{array}{l}\text { RISC-RNA } \\
\text { interaction } \\
\text { (requires } \\
\text { both the } \\
\text { miRNA and } \\
\text { the siRNA } \\
\text { pathways) }\end{array}$ & $\begin{array}{l}\text { Effective in } \\
\text { knockdown of } \\
\text { target genes with } \\
\text { ease of } \\
\text { multiplexing } \\
\text { atasiRNAs and } \\
\text { have fewer off- } \\
\text { target effects than } \\
\text { traditional RNAi. }\end{array}$ & $\begin{array}{l}\text { It is a knockdown } \\
\text { rather than a } \\
\text { knockout. Less } \\
\text { practiced than } \\
\text { amiRNAs in } \\
\text { publications. }\end{array}$ \\
\hline TM & $\begin{array}{l}\text { A long }(\sim 500 \mathrm{nt}) \text {, induced } \\
\text { phosphate starvation } 1 \\
(I P S 1) \text {-derived structures } \\
\text { having } 3 \text { central mismatches } \\
\text { at the miRNA binding site, } \\
\text { which serve as a target } \\
\text { mimic to sequester any } \\
\text { miRNA of interest for loss } \\
\text { of functions }\end{array}$ & $\begin{array}{l}\text { RISC-RNA } \\
\text { interaction } \\
\text { (Mechanism } \\
\text { not fully } \\
\text { understood) }\end{array}$ & $\begin{array}{l}\text { A simple } \\
\text { structure that } \\
\text { mainly sequesters } \\
\text { miRNAs for loss } \\
\text { of function. }\end{array}$ & $\begin{array}{l}\text { May not target } \\
\text { some high- } \\
\text { abundant } \\
\text { miRNAs (e.g. } \\
\text { miR166) for loss } \\
\text { of function, } \\
\text { leading to a } \\
\text { noticeable } \\
\text { phenotype. }\end{array}$ \\
\hline STTM & $\begin{array}{l}\text { A short ( } 100 \mathrm{nt}) \text {, artificial } \\
\text { non-coding RNA designed } \\
\text { to have two target mimics, } \\
\text { linked by a } 48-88 \mathrm{nt} \text { spacer, } \\
\text { having } 3 \text { central mismatches } \\
\text { at the miRNA binding sites } \\
\text { to trigger the degradation or }\end{array}$ & $\begin{array}{l}\text { RISC-RNA } \\
\text { interaction } \\
\text { (SDN- } \\
\text { mediated } \\
\text { miRNA } \\
\text { degradation, }\end{array}$ & $\begin{array}{l}\text { Mainly } \\
\text { knockdown the } \\
\text { target miRNA by } \\
\text { degrading and /or } \\
\text { sequestering it. }\end{array}$ & $\begin{array}{l}\text { May not target } \\
\text { low-abundant } \\
\text { miRNAs for loss } \\
\text { of function, } \\
\text { leading to a } \\
\text { noticeable } \\
\text { phenotype. }\end{array}$ \\
\hline
\end{tabular}




\begin{tabular}{|c|c|c|c|c|}
\hline & $\begin{array}{l}\text { sequestration of target } \\
\text { miRNAs. }\end{array}$ & $\begin{array}{c}\text { but } \\
\text { mechanism } \\
\text { not fully } \\
\text { understood) }\end{array}$ & & \\
\hline SP & $\begin{array}{l}\text { An artificial RNA having up } \\
\text { to } 15 \text { target mimics, } \\
\text { separated by a } 4 \text { nt spacer, } \\
\text { having } 2 \text { central mismatches } \\
\text { at the miRNA binding sites } \\
\text { to target any miRNA of } \\
\text { interest for loss of function. }\end{array}$ & $\begin{array}{c}\text { RISC-RNA } \\
\text { interaction } \\
\text { (Mechanism } \\
\text { not fully } \\
\text { understood) }\end{array}$ & $\begin{array}{l}\text { Powerful for } \\
\text { functional loss of } \\
\text { some miRNAs } \\
\text { that are not } \\
\text { effectively } \\
\text { knocked down by } \\
\text { both TMs and } \\
\text { STTMs }\end{array}$ & $\begin{array}{l}\text { Difficulty in } \\
\text { constructing a } \\
\text { long SP made of } \\
15 \text { tandem } \\
\text { repeats, separated } \\
\text { by } 4 \text { nt spacer. } 4 \\
\text { nt spacer limits } \\
\text { the application of } \\
\text { minimal SP such } \\
\text { as } 2 \text { tandem } \\
\text { repeats that is not } \\
\text { effective. }\end{array}$ \\
\hline CRISPR/Cas9 & $\begin{array}{l}\text { Contains a single guide } \\
\text { RNA (sgRNA) of 96-nt to } \\
\text { recognize and bind a } \\
\text { genomic DNA region of } 20 \\
\text { bp in a close proximity to } \\
\text { the PAM such as NGG. The } \\
\text { sgRNA has a 3' scaffold of } \\
76 \text { nt with a defined } \\
\text { secondary structure to } \\
\text { interact with the } \\
\text { endonuclease Cas9 for DNA } \\
\text { breakage and repairing. }\end{array}$ & $\begin{array}{l}\text { sgRNA/Cas9 } \\
\text {-DNA } \\
\text { interaction. } \\
\text { (DNA } \\
\text { cleavage and } \\
\text { repair) }\end{array}$ & $\begin{array}{l}\text { Most convenient } \\
\text { in targeting } \\
\text { gene/genome for } \\
\text { mutation, editing, } \\
\text { and regulation of } \\
\text { expression. }\end{array}$ & $\begin{array}{l}\text { Dependence upon } \\
\text { PAM (NGG) } \\
\text { sometimes makes } \\
\text { its use limited to } \\
\text { only a specific } \\
\text { region/and or } \\
\text { genes. Also } \\
\text { having off-target } \\
\text { effects genome- } \\
\text { wide. }\end{array}$ \\
\hline
\end{tabular}




\section{Outstanding Questions}

(1) What are the best dynamic interacting structures between miRNAs and miRNA target mimics for inactivating the functions of or triggering the degradation of miRNAs?

(2) What are the functions of endogenous miRNA backbones and how can they be better modified for production of highly potent amiRNAs?

(3) Is the current sgRNA scaffold the best for genome editing or can we modify it or screen for a better one for highly effective genome editing? 


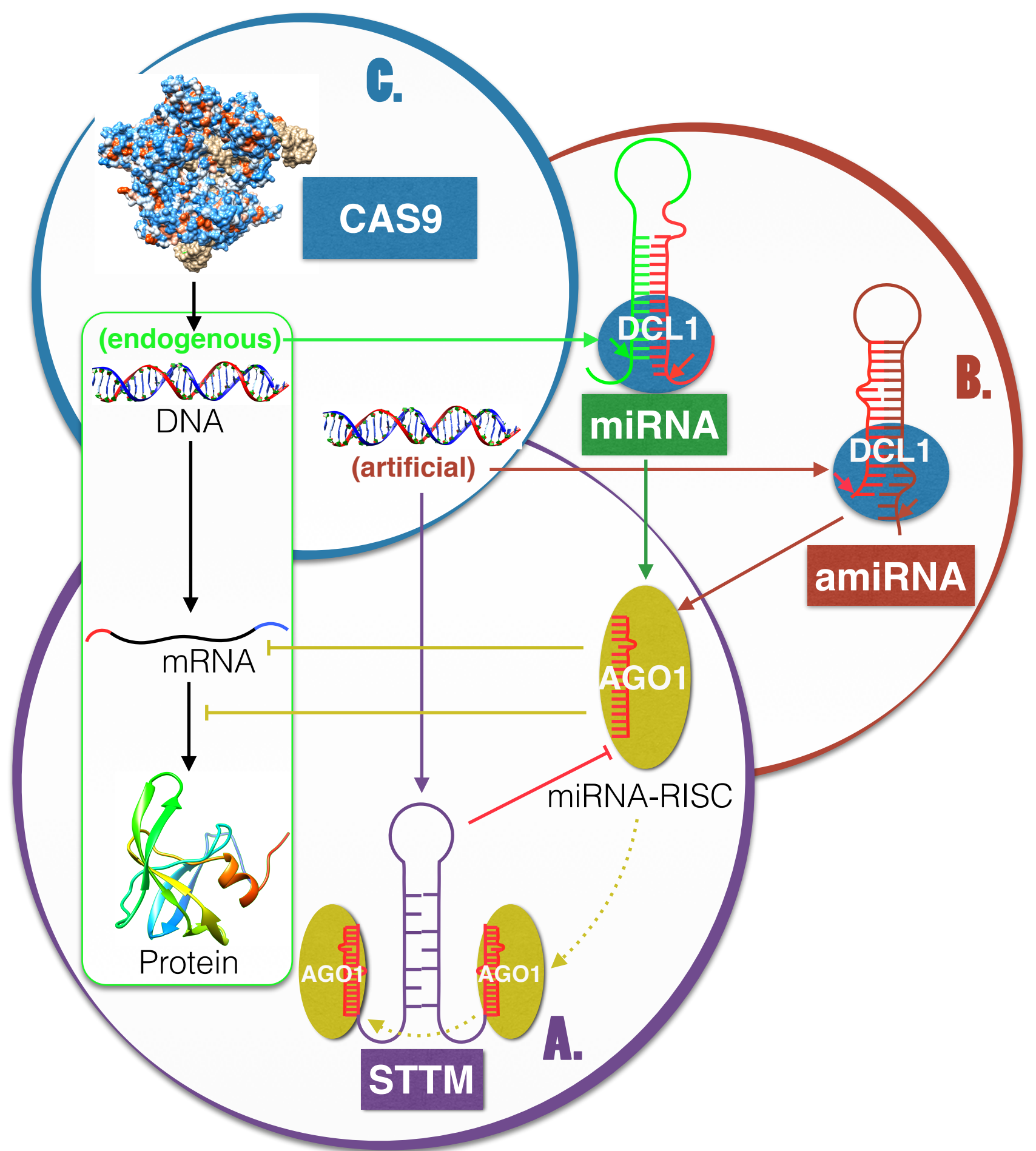




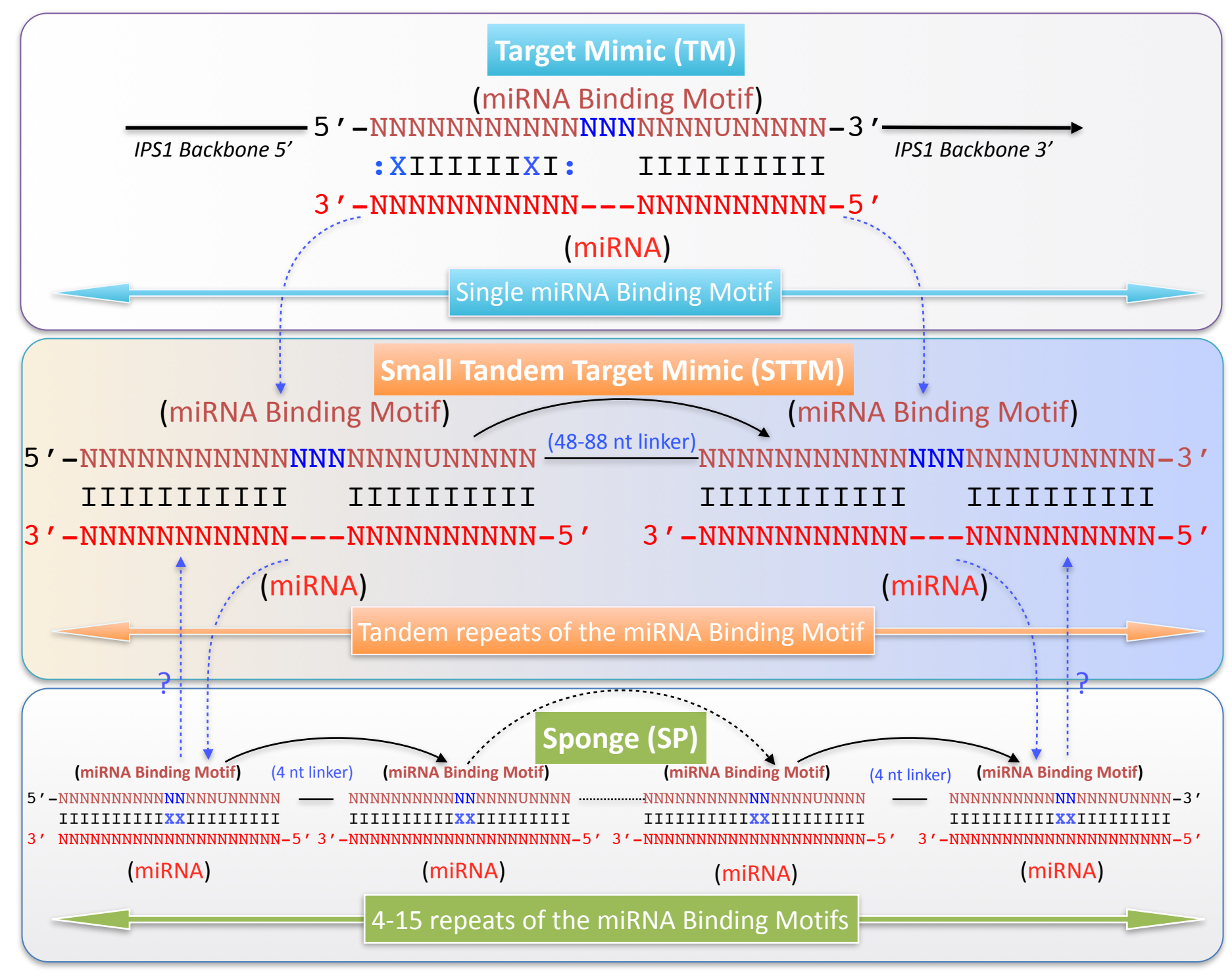




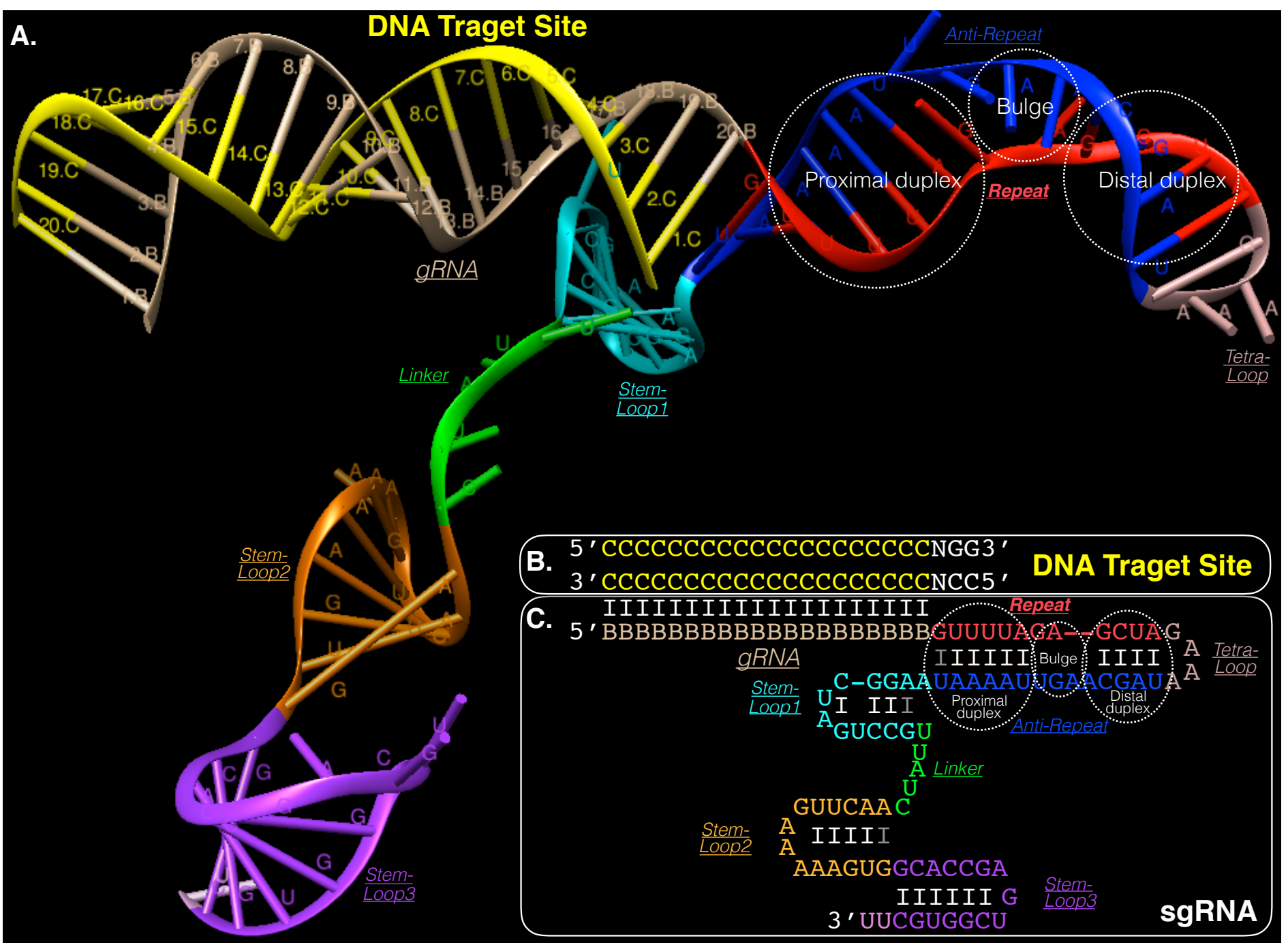




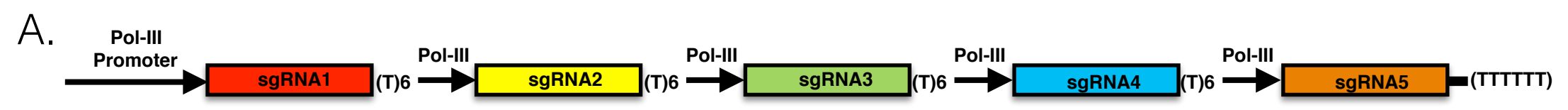

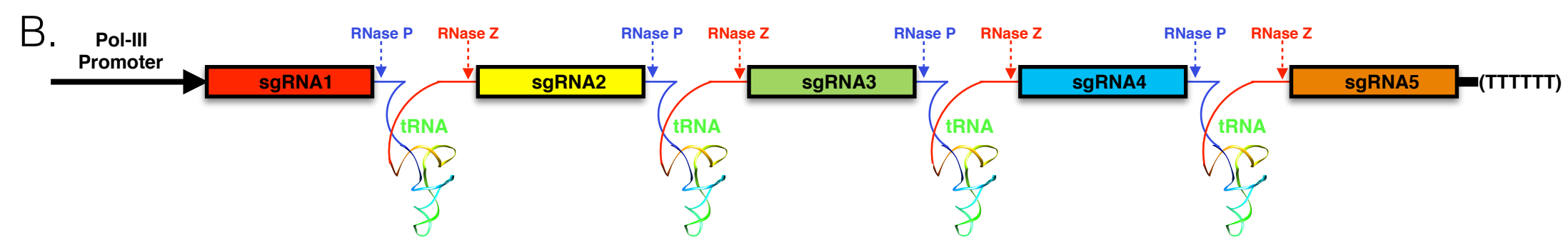

C. $\begin{gathered}\text { Pol-II HHRZ } \\ \text { Promoter }\end{gathered}$ 